\title{
A Data Collector for Route Inspection Based on Palm Handheld in Industry
}

\author{
Wang Jing ${ }^{1}$, Zhang Qing ${ }^{1}$, Zhang Sicong ${ }^{1}$, Liu Dan', and Xu Guanghua ${ }^{2}$ \\ 1. School of Mechanical Engineering, Xi'an Jiaotong University, Xi'an \\ 710049, P.R. China; \\ 2. State Key Laboratory for Manufacturing Systems Engineering, Xi'an \\ Jiaotong University, Xi'an 710049, P.R. China \\ Department of Instrument Science and Technology, School of Mechanical \\ Engineering, $X{ }^{\prime}$ 'an Jiaotong Unversity, $X i$ 'an 710049, P.R China
}

\begin{abstract}
Information management of equipments is a crucial component in enterprise resource plan (ERP) and data acquisition of equipment condition is the basis of information management system in enterprise. For most medium and small equipments, route inspection is the main method of condition information achievement. In this paper, condition information is divided into measurement information, display information and observation information. A data collector based on Palm handheld is developed to attain display and observation information. Acquired data is gathered to the route workstation in the spot. Then, each workstation uploads the data to the higher database server automatically, to share all the data in the range of whole enterprise. Results show that this data acquisition system enhances the efficiency of route inspection, provides support to discover the abnormity of machines and helps technicians to master the current condition of equipments timely.
\end{abstract}

\section{Introduction}

In process industry, condition information acquisition of equipments is crucial to realize predictive maintenance. Three methods, which are online system, portable system and route inspection, are utilized to acquire equipment condition data [1]. Compared with portable and online system, route inspection has more flexibility and lower cost. Thus, it is very propitious for the dispersive medium and small equipments.

Condition information of equipments is divided into measurement information, display information and observation information. Measurement information, such as acceleration, velocity and displacement, can be derived by handheld vibration data

Please use the following format when citing this chapter:

Jing, W., Qing, Z., Sicong, Z., Dan, L., Guanghua, X., 2006, in International Federation for Information Processing, Volume 205, Rescarch and Practical Issues of Enterprise Information Systems, eds. Tjoa, A.M., Xu, L., Chaudhry, S., (Boston:Springer), pp.489-498. 
collector, whereas in many factories, display information (pressure, voltage and current which are displayed on instruments) and observation information (description of measurement points) are recorded on paper, which is low efficiency. This goes against the management and utilization of equipment data. With the development of computer science, many data collectors for display and observation data acquisition are developed based on DSP or MCU. But these traditional data collectors got no extended application because of several key defects, which are:

a) Poor storage: most data collectors for display and observation information have a poor storage of less than $2 \mathrm{MB}$. It cannot meet the demand of millions of equipment data collection in large-scale enterprise.

b) Lack of input methods: only number input is supported. Chinese characters are supported restrictively. Some of them cannot display Chinese characters. Others support a Chinese character display, whereas do not hold a Chinese character input. This leads to a difficulty to record observation information, which is mainly composed of Chinese characters.

c) Portability: most data collectors for display and observation information are huge and clumsy. It is hard for workers to collect thousands of data by a jumbo.

With the development of communication science, efficient management and utilization of equipment data becomes possible. A route inspection system based on Internet called MTREE is quoted to support predictive maintenance of equipments which are numerous, various, isolated and dispersive. Technologies such as network, data communication, databases and equipment condition monitoring and fault diagnosis are used to realize the condition monitoring throughout life-span and management of mechatronic equipments. The Palm-based data collector presented in this paper is the main data achievement tool in MTREE. It accomplishes data acquisition of display information and observation information. Compared with traditional data collector for display and observation information, a Palm-based data collector has lots of advantages such as:

a) Abundant input methods: Chinese characters, English characters, numbers, symbols are supported. Users can input anything directly on the screen of the data collector by a special pen. It is as convenient as you record the information on a piece of paper. Traditional data collector for display and observation information only provides a few of buttons on it, which supports simple operations or English character input.

b) Strong memory extension ability: we develop a data acquisition system based on Zire71 Palm handheld. It has 16M RAM and a SD extension slot. Storage can be extended to $4 \mathrm{G}$, which can hold trillions of data. It is unnecessary for technicians to upload data to workstation frequently anymore, just because there is no more memory to save more equipment condition data. 
Multiple communication interfaces: USB, infrared and serial communication are supported. Data transfer between workstation (PC) and data collector (Palm handheld) becomes much faster than ever.

\section{Data collector in MTREE}

MTREE, an information management system, is constructed based on database in enterprise intranet. It is the platform for implementing information management of equipment condition in enterprise. All route inspection data of equipment condition in different departments is uploaded to the database server. Thus, all the information is shared in the entire factory. By the equipment condition data, managers can master the equipment running state and discover abnormity timely, which supports predictive maintenance and optimizes equipment management. The framework of MTREE is shown in Fig. 1.

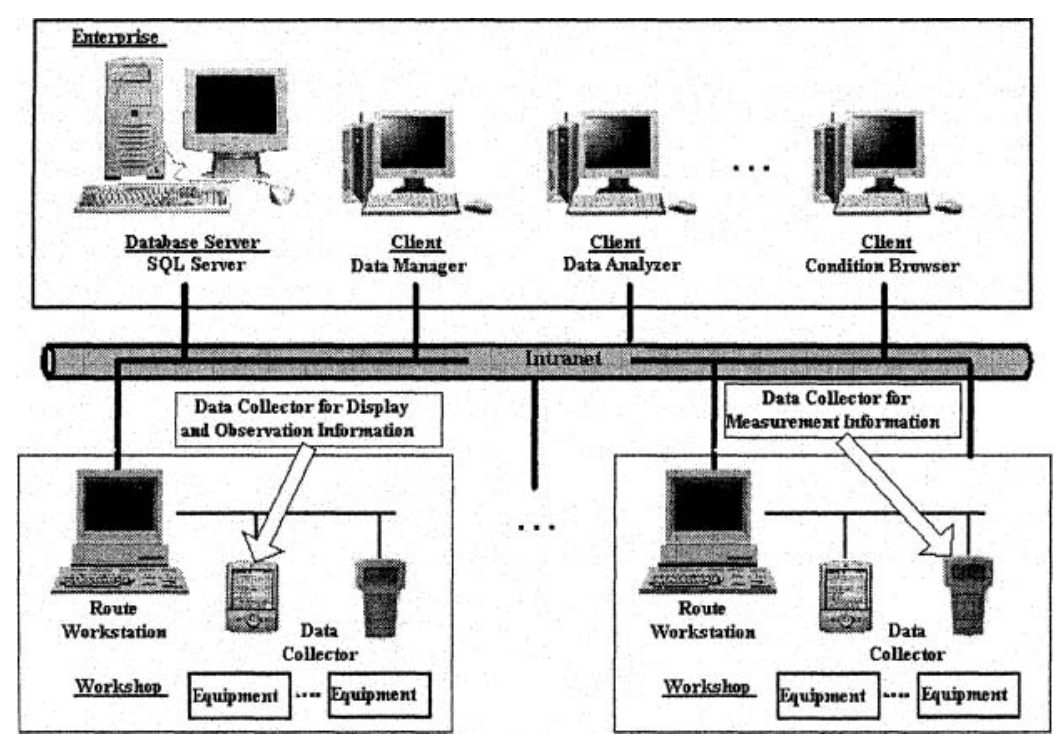

Fig. 1. Framework of MTREE

Equipment condition data is the key support of the route inspection system (MTREE). Moreover, there are a lot of display and observation information in route inspection information. So, a data collector for display and observation information is a sticking point in the route inspection system. It should: a) be convenient to data acquisition and upload; b) have good portability and a large memory. Palm handheld owns the basic hardware requirement that is portable 
and easy to extend memory. Data upload can be expediently achieved by its strong communication ability. Also, since users can input something directly on the screen of Palm handheld, acquisition of display and observation information becomes much easier. Thus, a data collector for display and observation information is developed based on Palm handheld in the route inspection system (MTREE). The corresponding software is designed for data acquisition, uploading on the Palm side, and data management on the workstation side.

\section{Structure of data collector system for display and observation information}

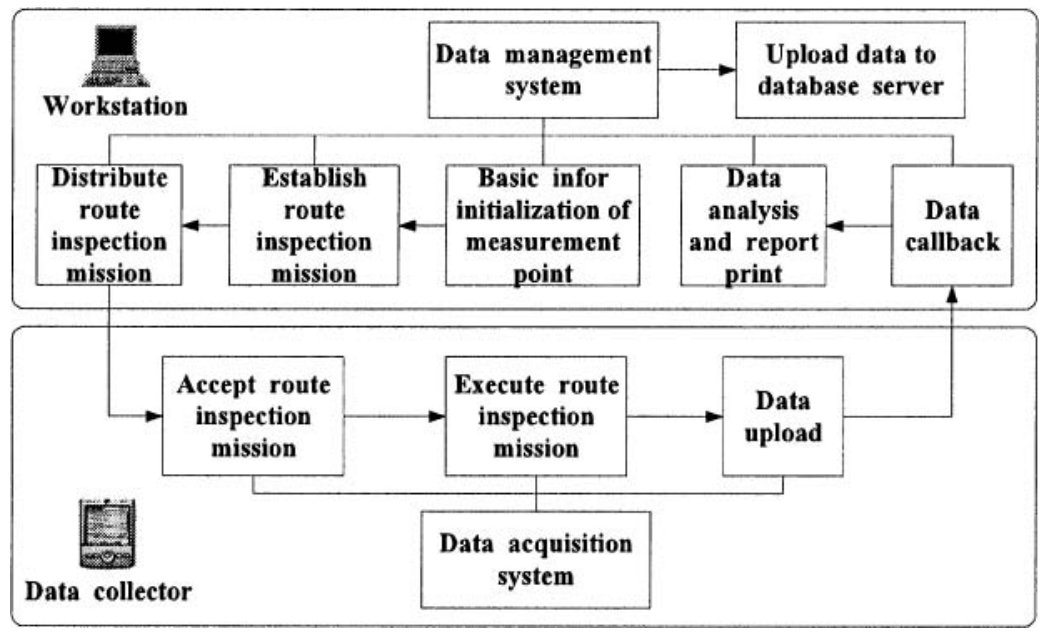

Fig. 2. Structure of data collector system for display and observation information

According to the characteristics of route inspection information for equipment condition, display and observation information are acquired dispersedly and supervised uniformly. The structure of data collector for display and observation information system is shown in Fig. 2.

The data collector system for display and observation information is constituted of data acquisition system on data collector and data management system on workstation. Data collector achieves data acquisition of equipment condition and workstation focuses on data management and utilization. USB port is used to realize data exchange between data collector and workstation. Whole system works as the following steps: First of all, by the database management tool in the data management system in workstation, route inspection manager inputs all the basic 
information of measurement points, such as department information, equipment number, measurement point type, to system database. Then, route inspection missions are established. After that, necessary missions are transferred to data collector, using USB communication. Inspectors take the data collector with missions to worksite and find the corresponding measurement point in the route inspection mission. For instance, measurement point $\mathrm{A}$ is a current of ammeter in department $B$ with equipment number $C$. The inspector finds the ammeter in the stated place and record the current of the ammeter to data collector. When all the necessary missions are completed, the inspector uploads the collected data to the nearest workstation. Route inspection manager analyzes the data to decide whether the equipment is in good condition or not. Reports of equipments, measurement points and inspected data are printed if necessary. At the same time, collected data on different workstation are uploaded to the database server automatically where all the data are stored and shared by the intranet in enterprise.

Since the different characteristics of display information and observation information, different acquisition patterns are used to them. Measurement points of display information include the following contents: 1) the department name, equipment number and measurement point number that is unique to a measurement point; 2) measurement point type (such as velocity, temperature, flux, etc), unit dimension (such as $\mathrm{mm} / \mathrm{s},{ }^{\circ} \mathrm{C}, \mathrm{M}^{3} / \mathrm{h}$ ), reference value, maximum value and minimum value. Inspectors are asked to record the displayed value on the instrument (such as 1 $\mathrm{mA}$ on an ammeter) to the data collector. Reference value, maximum value and minimum value are used to estimate whether collected data is normal or abnormal. Measurement points of observation information include the following contents: 1) the department name, equipment number and measurement point number that is unique to a measurement point; 2) probable description of the measurement point (for a shaft, it could be "normal", "misalignment", "wear", "damaged", "in repair") are set in the description database. Inspectors decide the condition of the measurement point by a certain tool and select the appropriate description for it on data collector. If there is no appropriate description in the selection, an "unknown" description can be selected as the temporary option. After all the data are uploaded to the workstation, a new description will be added to the description database.

4 Key technologies

Data management system on workstation is developed by Visual Basic 6.0. System database is designed in Access 2000. Following functions are supported in the system: initialization of measurement point information, establishment and distribution of route inspection mission, callback of equipment condition data, inquiring and editing, data trend analysis of equipment condition data, and building correlated reports. Data acquisition system on data collector is developed by CodeWarrior 9.0, programmed in C language. Operations listed below are supported in the system: Acceptance of route inspection mission, periodical route inspection, data collection, inquiry, modification and upload, statistics on execution of route inspection mission and clue to illegal data. 


\subsection{Database design}

The database file on route collector includes all the information of route inspection mission. It is a binary file with an extension PDB (Palm Database). A PDB file is composed of the following three parts [2]:

- Header. The first 78 bytes of PDB file is called file header, which includes the following information: database name, flags, version, create time, modified time, backup time, modified number, application information size, sort information size, type, creator ID, unique ID seed, next record list and number of records;

- Record Entry Index. Record entry index starts from 79th bytes. Each index for a record takes 8 bytes. It is like table of contents in a book. The position (offset) of a record can be found by its entry index.

- Record Data. Record data follows record entry index. All the information of measurement points is included in record data. This is the main body of the database.

A record in the database is composed of all the information of one measurement point in the route inspection mission. Data expressions of display and observation information are different. Display information is expressed in number, whereas observation information is expressed in characters. They are crisscross in the route inspection mission. In order to enhance the search speed for a record in the database, display and observation information are stored in one database with a mark "measurement point type" for differentiation. A record in the route inspection mission database includes the following contents:

Common information. Common information exists in both display and observation measurement point, which comprises route inspection mission name, department name, equipment number, measurement point number and measurement point type. A record can be uniquely indexed by its route inspection mission name, department name, equipment number and measurement point number. Measurement point type specifies the measurement point is a display or observation point.

Unique information in display measurement points. Unique information in display measurement points includes record value, maximum value, minimum value, reference value, and unit dimension. Inspectors are asked to record the displayed value on the instrument as "record value". Maximum value and minimum value indicates whether the record value is normal. Reference value gives the standard of the measurement point value. Unit dimension includes volt-unit of voltage, ampere-unit of current, etc.

Unique information in observation measurement points. Unique information in observation measurement points includes description of measurement points, which are set in the description database. Inspectors select the appropriate description for observation measurement points on data collector. 


\subsection{Communication module design}

Communication module achieves the data exchange between workstation and data collector. A communication cradle is used to connect Palm handheld (data collector) with USB port on PC (workstation). On data collector, Palm OS serial communications stack is used to finish file transfer between Palm handheld and PC. On workstation side (PC), a DLL for USB file transfer provided by Palm is utilized.

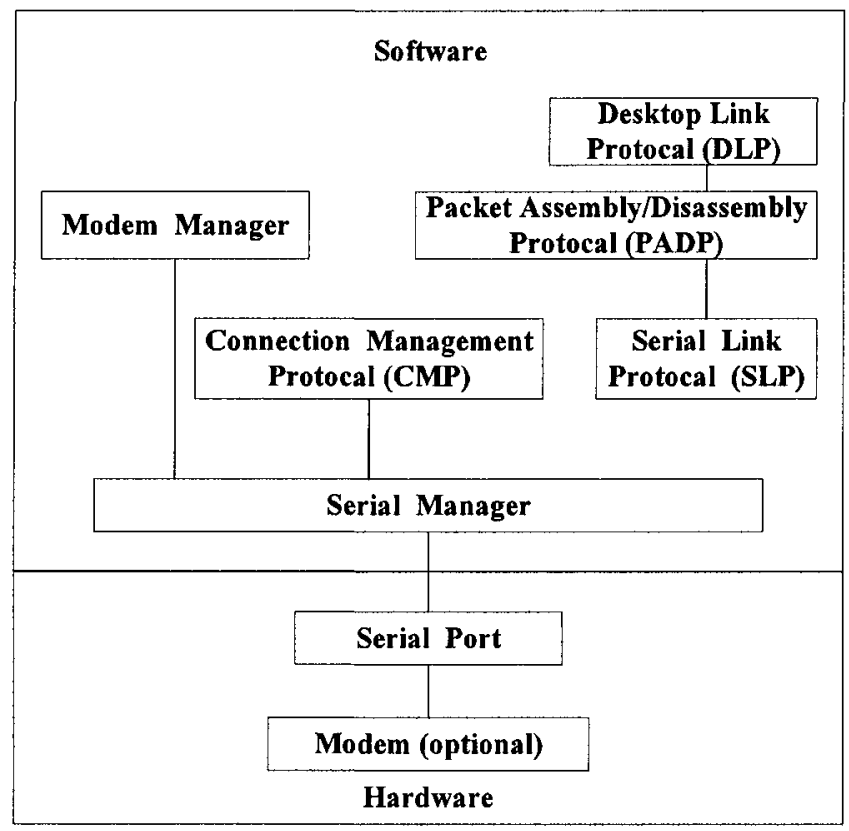

Fig. 3. Layers that make up the Palm OS serial communications stack

The Palm OS serial port supports serial communications at speeds between 300 and $115,200 \mathrm{bps}$. On the software side, the Palm OS has several layers that make up its serial communications system [3]. Each layer adds to and relies upon the capabilities of the layer beneath it. Fig. 3 shows how the different layers of the Palm OS serial communications stack relate to one another.

Serial manager. At the lowest level, the serial manager provides direct control of RS-232 signals and the hardware serial port. This layer allows for byte-level serial input and output, which makes this layer the most flexible for use in custom applications.

Modem manager. Built directly on top of the serial manager, the modem manager provides a small API for modem dialing and control, which is capable of 
handling a modem attached either directly to the handheld's serial port or through a Palm modem cable.

Serial Link Protocol (SLP). Also built on the serial manager, this protocol provides an efficient send-and-receive system for data packets, including CRC-16 error checking. Both the HotSync desktop program and the Palm Debugger use this protocol for communicating with a Palm OS handheld resting in its cradle.

Packet Assembly/Disassembly Protocol (PADP). Built on the Serial Link Protocol, PADP provides buffered data transmission capabilities for the Desktop Link Protocol, described below. PADP is entirely internal, and your applications do not have access to this layer.

Desktop Link Protocol (DLP). DLP is built on top of PADP and provides remote access to various Palm OS subsystems, including data storage. HotSync technology uses DLP to perform synchronization and to install and back up databases. Though you cannot directly access DLP's features through a Palm OS application, you indirectly make use of it if you write a HotSync conduit for a desktop computer.

Connection Management Protocol (CMP). CMP is built directly on the serial manager layer, and it is another protocol the system uses for negotiating baud rates and exchanging basic information with outside communication software. Only the operating system has access to CMP.

Since serial manager allows for byte-level serial input and output, it makes this layer the most flexible for use in custom applications. Therefore, serial manager is selected as the solution of communication module on data collector.

\section{Realization of data collector system for display and observation information}

Data collector system for display and observation information is divided into data acquisition system on data collector and data management system on workstation. The main interface of data management system on workstation is shown in Fig. 4, which includes the following modules:

1) Establishment, Distribution and callback of route inspection mission. In the establishment of route inspection mission, add, modification and deletion of route inspection missions or measurement points in route inspection mission are allowed by administrator of the system. Inspectors can download certain route inspection missions to data collector. When the mission is done, collected data is uploaded to workstation.

2) Analysis of route inspection data, Report center and equipment running $\log$. Analysis of route inspection data makes a trend analysis of certain measurement point data on equipment. In report center, reports listed below can be printed or written in Microsoft Word documents: equipment report, measurement point report, route inspection data report, route 
inspection mission report, equipment running log report, statistics report of equipment running.

3) Database maintenance tool. System administrator is allowed to create and maintain route inspection database, using database maintenance tool. The following operations are supported:

- Create a new route inspection database;

- Maintain existed databases;

- Data inquiry;

- Other tools, including database upgrade and combination, configuration of combination, database backup and clearance, editing description of observation information.

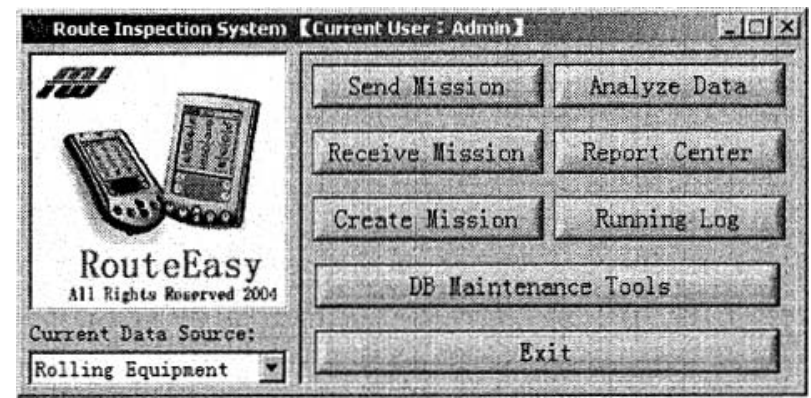

Fig. 4. Main interface of data management system on workstation

Data acquisition system on data collector includes the following functions:

1) Data acquisition: including data acquisition for display information and observation information. Typical data acquisition interfaces for display and observation information are shown in Fig. 5 (a) and (b);

2) Statistics on execution of route inspection missions: Statistics on execution of current route inspection mission, which includes measurement point state (whether the point is executed) and route inspection time (information input time), as shown in Fig. 5 (c). 


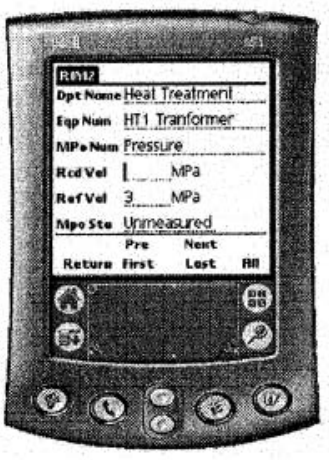

(a)

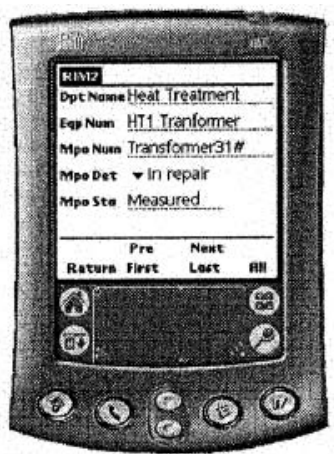

(b)

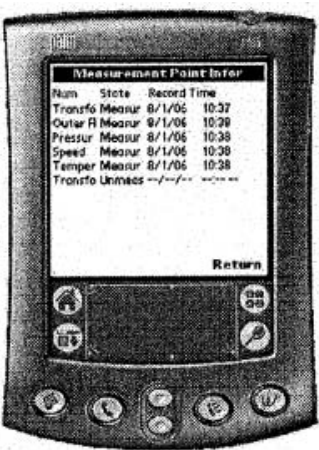

(c)

Fig. 5. Interfaces in route inspection system for display and observation information. (a) Data acquisition interface for display information; (b) data acquisition interface for observation information; (c) Statistics on execution of route inspection missions

\section{Conclusions}

Route inspection system for display and observation information presented in this paper, has been used in a steel rolling factory in Wuhan, P.R. China, since 2004. Results show that all the functions in the system work steadily. Compared with the traditional data collector for display and observation information, Palm Handheld based data collector for display and observation information enhances the efficiency and precision of route inspection for display and observation information, which supports predictive maintenance of equipments.

\section{References}

1. M. Xu, Equipment Fault Diagnosis Manual (Xi'an Jiaotong University Press, Xi'an, 1998) 2. S. Mann and R. Rischapter. Advanced Palm Programming (China Machine Press, Bejing, 2002)

3. L.R. Foster. Palm OS Programming Bible (IDG Books Worldwide, Inc., Foster City, 2000) 